\title{
Molecular Relaxations of a Branched Poly(oxyethylene) Network Polymer
}

\author{
Kimio ICHIKaWA, ${ }^{\dagger}$ L. Charles Dickinson, ${ }^{*}$ William J. MaCKNIGHT, ${ }^{*}$ \\ and Masayoshi WatanabE** \\ Fujinomiya Lab. Fuji Photo Film Co., Ltd., 200 Onakazato, Fujinomiya, \\ Shizuoka 418, Japan \\ * Polymer Science and Engineering Department, University of Massachusetts, \\ Amherst, MA 01003, U.S.A. \\ ** Department of Chemistry, Yokohama National University, Tokiwadai, \\ Hodogaya-ku, Yokohama 240, Japan
}

(Received September 27, 1996)

\begin{abstract}
Dielectric relaxation measurements and ${ }^{13} \mathrm{C}$ NMR measurements were carried out on an amorphous network polymer derived from poly[2-(2-methoxyethoxy)ethyl glycidyl ether]triol with 2,4-toluenediisocyanate. The main relaxation and the higher frequency relaxation were observed in the dielectric spectra. The main relaxation was found to be symmetrical and broad with a half width of 5 decades in a frequency domain. The higher frequency process is considered to be the $\gamma$ process of ethylene oxide chains due to the local twisting mode. The temperature dependence of $T_{1}$ and $T_{1 \rho}$ of the ethylene oxide carbon showed minima and it is clearly shown that the relaxation times obtained from the dielectric measurements correspond to the relaxation time obtained from the $T_{1 \rho}$ measurements of the ethylene oxide carbon. The temperature dependence of $T_{1}$ was well described assuming the Cole-Cole spectral density function with the shape factor $\beta$ obtained for the dielectric main relaxation. The ${ }^{13} \mathrm{C}$ magnetization decay of the ethylene oxide carbon in the rotating frame was found to be given by two components (slow and fast components) below the relaxation temperature. The broad dielectric relaxation and the decay profiles of the ethylene oxide carbon are discussed in terms of the branched structure of the network.

KEY WORDS Branched Network Polymer / Dielectric Relaxation / ${ }^{13} \mathrm{C}$ Nuclear Magnetic Resonance /

Dynamic Mechanical Analysis / Main Chain Motion / Side Chain Motion /
\end{abstract}

Amorphous network polymers of poly(ether polyol)s crosslinked with isocyanates have been received considerable interest as matrix polymers of solid polymer electrolytes due to their advantages in thermal and mechanical properties. ${ }^{1,2}$ The poly(ethylene oxide) (PEO) based networks with branched polyether chains were prepared and found to show the highest conductivity in the solvent-free polymer electrolytes. ${ }^{3,4}$ It has been suggested on the ionic conductivity mechanism of this particular electrolyte that the side chain motions would contribute to the high conductivity.

From a more fundamental point of view, the introduction of the side chain groups could be expected to introduce different modes of molecular motions in networks, however the molecular motions in these network polymers have not yet been investigated.

Molecular motions of network polymers derived from poly(propylene oxide)s ( $\mathrm{PPO}$ ) with a trifunctional isocyanate have been investigated by solid state ${ }^{13} \mathrm{C}$ NMR techniques ${ }^{5,6}$ and dielectric relaxation studies, ${ }^{7}$ it has been found that the relaxation time of the back bone ether residue is shorter than that of the cross link point (urethane unit). The ionic conduction behavior of these network polymers with lithium perchlorate were investigated using ${ }^{7} \mathrm{Li}-\mathrm{NMR}$, mechanical measurements, and FT-RAMAN spectroscopy, and it was found that the ionic motions observed were considered to be coupled with motions due to backbone ether residue motions and urethane unit motions. ${ }^{8-10}$

In the present paper, the molecular motions of a poly(ethylene oxide) based network with a branched side chain structure are investigated using dielectric measure-

\footnotetext{
† To whom correspondence should be addressed.
}

ments and magic-angle spinning with direct polarization ${ }^{13} \mathrm{C}$ NMR (MAS-DP-NMR) and discussed in terms of the branched structure.

\section{EXPERIMENTAL}

The network polymer was obtained by crosslinking poly[2-(2-methoxyethoxy)ethyl glycidyl ether]triol (nominal molecular weight 5350) with 2,4-toluenediisocyanate under a stoichiometric condition. The details on preparation procedure have been given elsewhere. ${ }^{3}$

Films for dielectric measurements with uniform thickness of $0.4 \mathrm{~mm}$ were cut into disks of $33 \mathrm{~mm}$ diameter, and $\mathrm{Au}$ electrodes were coated onto both surface of the disks. Dielectric measurements were carried out using a DETA mk-II (Polymer Laboratories, Ltd.) in the frequency range of $66 \mathrm{~Hz}$ to $100 \mathrm{kHz}$ from $-90^{\circ} \mathrm{C}$ to $-40^{\circ} \mathrm{C}$ under the nitrogen atmosphere.

${ }^{13} \mathrm{C}$ NMR measurements were carried out using an IBM 200 A C Spectrometer with solids accessory at $50.3 \mathrm{MHz}$ for ${ }^{13} \mathrm{C}$. All spectra were obtained with spinning a sample at $4 \mathrm{kHz}$ and with high power proton decoupling. $T_{1}$ was determined by the standard $\pi-\tau-\pi / 2$ sequence, and $T_{1 \rho}$ was determined as the decay of ${ }^{13} \mathrm{C}$ magnetization during spin lock of length $0.1-15 \mathrm{~ms}$ after a single $\pi / 2$ preparation pulse. The spin lock power was $50 \mathrm{kHz}$, making the proton and carbon $\pi / 2$ lengths $5 \mu \mathrm{s}$.

DSC measurements were carried out with a PerkinElmer DSC-4 over the temperature range of $-100^{\circ} \mathrm{C}$ to $50{ }^{\circ} \mathrm{C}$ at a heating rate of $20^{\circ} \mathrm{C} \mathrm{min}^{-1}$. The glass transition temperature $T_{\mathrm{g}}$ was defined as the midpoint of the heat capacity change.

Dynamic mechanical bending mode spectra with the temperature range of $-120^{\circ} \mathrm{C}$ to $20^{\circ} \mathrm{C}$ were carried out 
with a Polymer Laboratories DMTA at $0.33 \mathrm{~Hz}$ to $10 \mathrm{~Hz}$ with the heating rate of $1^{\circ} \mathrm{Cmin}^{-1}$ under the nitrogen atmosphere.

The samples were dryed at $80^{\circ} \mathrm{C}$ in vacuo for 2 days before above measurements. The samples were clear and transparent and DSC thermograph showed single glass transition temperature at $-68^{\circ} \mathrm{C}$. FT-IR spectra showed no residual isocyanate peak and hydroxyl peak.

\section{RESULTS AND DISCUSSION}

\section{Relaxation Data}

The Cole-Cole plots of the complex permittivity at $-90^{\circ} \mathrm{C}$ to $-40^{\circ} \mathrm{C}$ are shown in Figure 1 . There can be clearly seen the main broad dispersion with the estimated relaxation strength of 10 to 12 . As is seen in the plots for $-60^{\circ} \mathrm{C}$ and $-70^{\circ} \mathrm{C}$, a small secondary process can be recognized and the approximate position is indicated in the figure. Figure 2 shows the normalized curve of the imaginary part of the complex permittivity and the half width is 5 decades in the frequency domain. No temperature dependence of the shape of this main relaxation curve is also seen in Figure 2.

DMTA spectra obtained at $1 \mathrm{~Hz}$ at the temperature range from $-120^{\circ} \mathrm{C}$ to $20^{\circ} \mathrm{C}$ showed one transition peak at $-50^{\circ} \mathrm{C}$ as shown in Figure 3. A DSC profile of

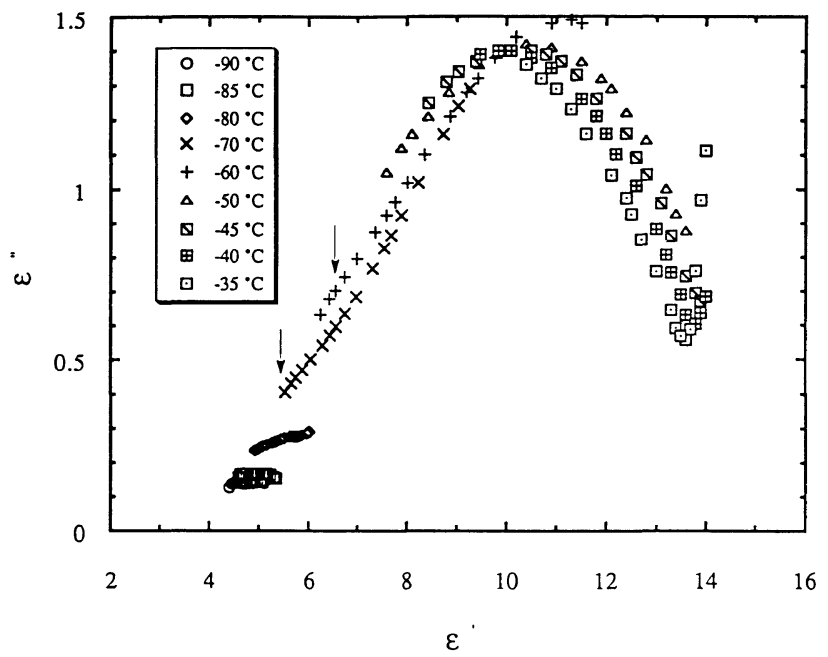

Figure 1. Cole-Cole plots of the complex permittivity obtained at various temperatures. Arrows indicate positions of the secondary process.

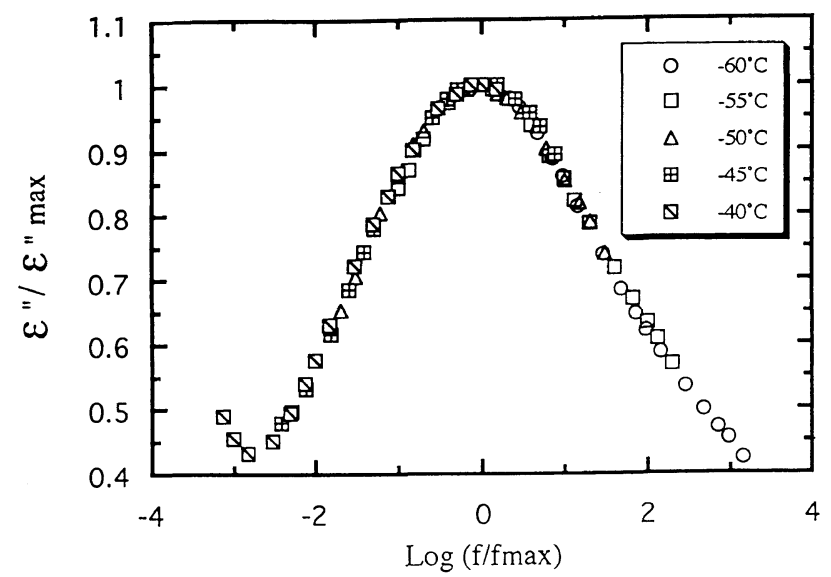

Figure 2. Normalized dielectric constants $\varepsilon^{\prime \prime}$ as a function of frequency at various temperatures. the network showed a single $T_{\mathrm{g}}$ at $-57^{\circ} \mathrm{C}$ defined as a midpoint of the heat capacity change. Therefore the present network polymer could be considered to be amorphous.

The ${ }^{13} \mathrm{C}$ NMR spectra of the network at room temperature are shown in Figure 4 and the peak assignments are shown. Figure 5 shows the decay profiles

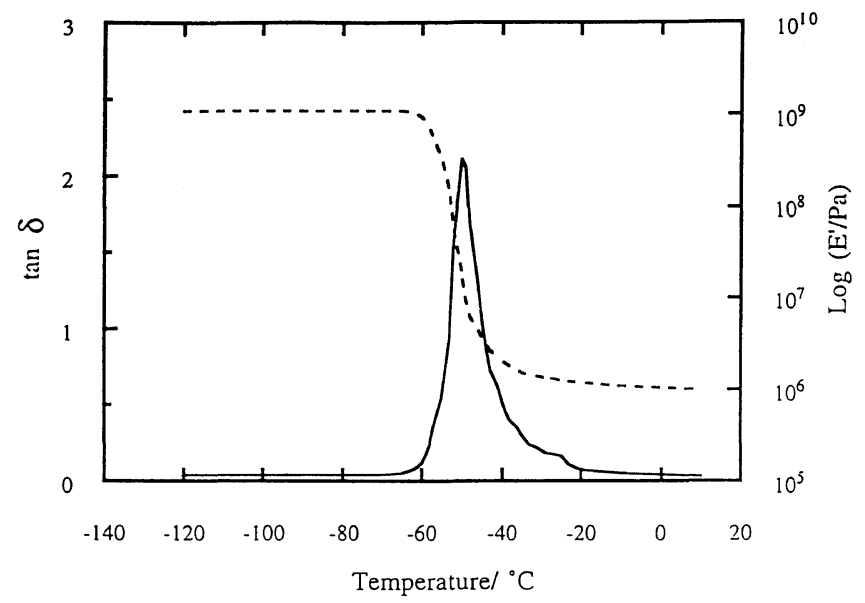

Figure 3. Dynamic mechanical spectra obtained at $1 \mathrm{~Hz}$ as a function of temperature.

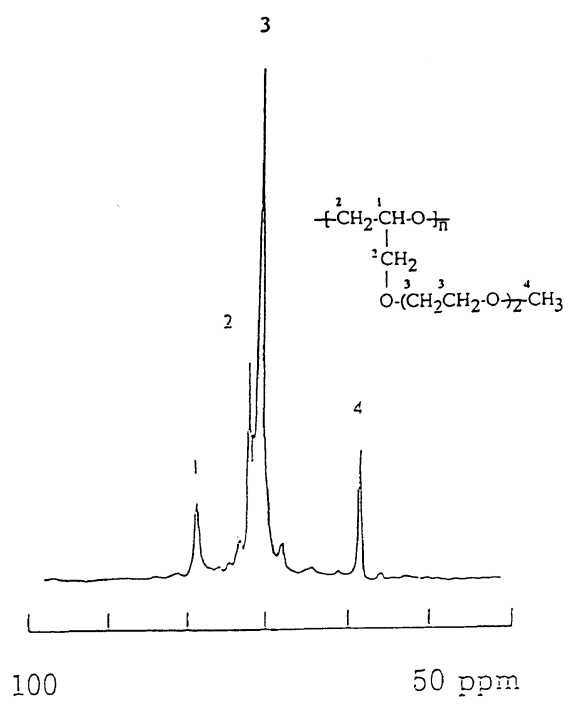

$\delta$ from TMS

Figure 4. ${ }^{13} \mathrm{C}$ MAS NMR spectra obtained at room temperature. Peak assignments are $1_{(78.6 \mathrm{ppm})},-\mathrm{OCH}-; 2_{(71.9 \mathrm{ppm})}, \mathrm{CH}-\mathrm{CH}_{2} \mathrm{O}-$; $3_{(70.4 \mathrm{ppm})},-\mathrm{OCH}_{2} \mathrm{CH}_{2}-; 4_{(58.4 \mathrm{ppm})},-\mathrm{OCH}_{3}$.



Figure 5. Decay profiles of the ethylene oxide carbon magnetization. 




Figure 6. Temperature dependences of fractions for individual components: $\triangle$, fast component; $\bigcirc$, slow component.



Figure 7. Temperature dependences of ${ }^{13} \mathrm{C} T_{1}$ of the ethylene oxide carbon: $O$, observed; ---, calculated.

of the ethylene oxide carbon (carbon 3) in the rotating frame and at temperatures below $-50^{\circ} \mathrm{C}$ the decay profiles were found to be biphasic. The fraction of each component was estimated by intersects of the magnetization. The fraction of the fast component was estimated to be 0.36 at $-80^{\circ} \mathrm{C}$ and increased with increasing the temperature and then reaches unity (single exponential decay) at $-40^{\circ} \mathrm{C}$. The fraction of the slow component was 0.69 at $-80^{\circ} \mathrm{C}$ and then decreased with increasing the temperature as shown in Figure 6. Decay profiles of the ethylene oxide carbon magnetization in $T_{1}$ measurements were single exponential in the temperature range from $-50^{\circ} \mathrm{C}$ to $25^{\circ} \mathrm{C}$. The temperature dependence of $T_{1}$ of the present sample is shown in Figure 7, and it could be expressed assuming broad distributions of correlation times with a mean value of them. Details are mentioned in the next section.

The relaxation times (in frequencies) obtained in the present study are plotted in Figure 8 . The relaxation times for the $\gamma$ relaxation $^{11}$ and the higher frequency relaxation observed in the present study are plotted in the figure. Since the $T_{1}$ and $T_{1 \rho}$ measurements were carried out at $50 \mathrm{MHz}$ and $50 \mathrm{kHz}$ respectively, the temperatures which give the minimum $T_{1}$ and $T_{1 \rho}$ was considered as relaxation temperatures. It is clearly demonstrated that the dielectric main relaxation observed is due to the ethylene oxide motions. The relaxation times $\tau_{\mathrm{c}}$ obtained by NMR and dielectric measurements could

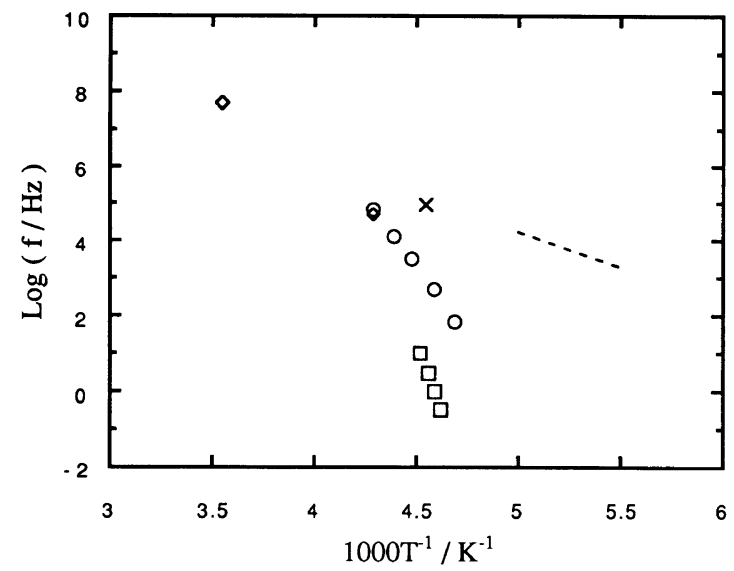

Figure 8. Relaxation times obtained at the present study: $\bigcirc$, dielectric main relaxation; $\diamond,{ }^{13} \mathrm{C} N \mathrm{NMR}$ relaxation of ethylene oxide carbon; $\square$, dynamic mechanical spectra; $\times$, dielectric higher frequency relaxation; ---, $\gamma$-relaxation of PEO. ${ }^{11}$

be discussed on the basis of Vogel-Fulcher-Tammann (VFT) equation. ${ }^{12}$

$$
\tau_{\mathrm{c}}=\tau_{0} \exp \left[B /\left(T-T_{0}\right)\right]
$$

where $\tau_{0}=9.52 \times 10^{-13} \mathrm{~s}, B=1159.2 \mathrm{~K}, T_{0}=163 \mathrm{~K}$ to obtain the best fitted result for both NMR and dielectric data.

The relaxation frequencies obtained by DMTA measurements are smaller than those of dielectric measurements. A correspondence between dielectric and mechanical relaxation can be expected when the molecular motions responsible for a mechanical relaxation involve reorientation of a polar group. Even in this case, it is reasonable to consider that the apparent activation energies obtained by mechanical measurements could be larger than those obtained by dielectric measurements because of the so-called internal friction effects, and such effects make the mechanical relaxation frequencies lower than the dielectric relaxation frequencies.

PEO's are known to show three relaxation processes; $\alpha, \beta$, and $\gamma$ processes. ${ }^{11}$ The $\alpha$ process is associated with the crystalline phase, and PEO crystallizes in a helical form and the melting point of this phase is observed in the temperature range from $60^{\circ} \mathrm{C}$ to $65^{\circ} \mathrm{C}$ for the high molecular weight PEO's. However, the $\alpha$ relaxation is not observed in dielectric spectra because of the large rising loss due to conductivity effects. The dielectric $\beta$ relaxation in PEO is related to the amorphous glass transition in disordered regions and the $T_{\mathrm{g}}$ 's depend on the crystallinity for high molecular weight PEO's. The $\gamma$ process observed around $-100^{\circ} \mathrm{C}$ at $1 \mathrm{kHz}$ with the apparent activation energy of 30 to $40 \mathrm{kcal} \mathrm{mol}^{-1}$ is considered to be motions associated with the local twisting motion of main chains. Since the present network is considered to be one phase amorphous structure on the basis of DSC and mechanical results, the dielectric main relaxation is due to the glass transition of the network. ${ }^{13} \mathrm{C}$ NMR results are also consistent with the dielectric results.

The broad distribution with the half width of 5 decades of the main dielectric dispersion is one of the characteristic features and the NMR decay profiles suggest contributions of the side chain structure to the 

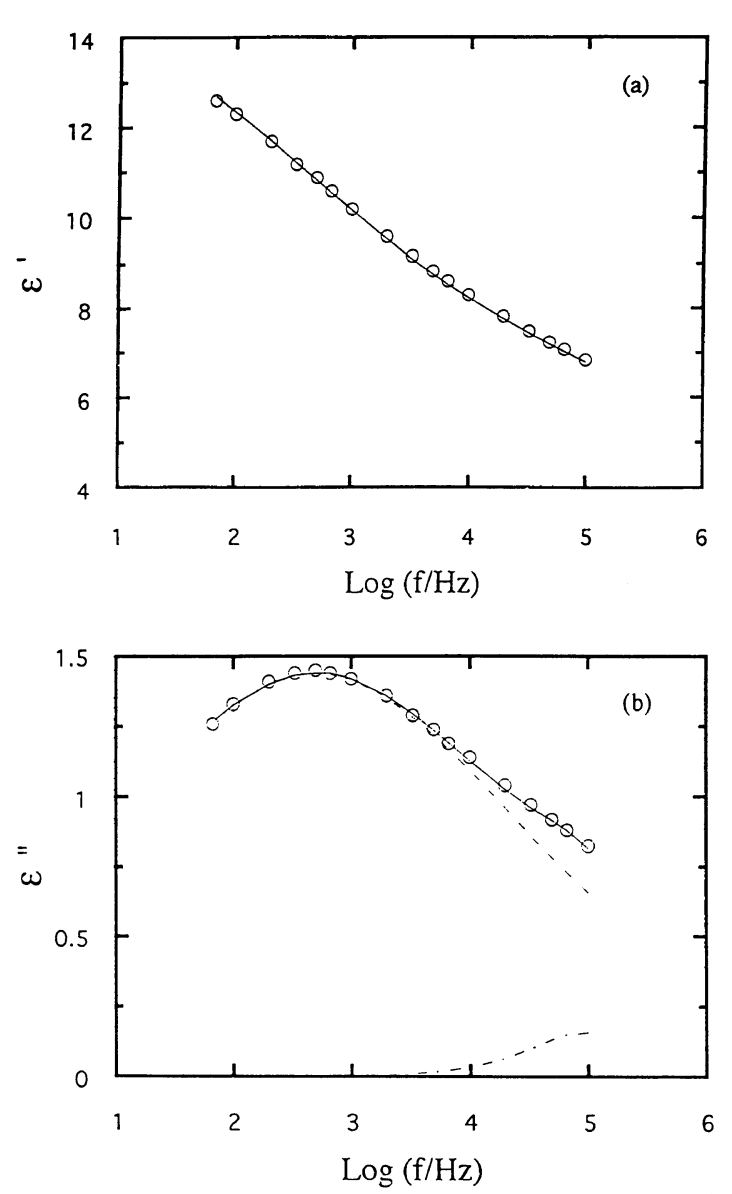

Figure 9. Frequency dependences of (a) $\varepsilon^{\prime}$ and (b) $\varepsilon^{\prime \prime}$ at $-55^{\circ} \mathrm{C}$. Solid lines and broken lines are those calculated from eq 2 .

dielectric main dispersion. The relaxation strength was estimated to be 10 to 12 from the Cole-Cole plots in the present case. In order to discuss the dielectric parameters qualitatively, the complex permittivity was assumed to be the sum of two Cole-Cole contributions ${ }^{13}$;

$$
\varepsilon^{*}(\omega)=\varepsilon_{\infty}+\sum \Delta \varepsilon_{j} /\left[1+\left(i \omega \tau_{j}\right) \beta_{j}\right] \quad j=h, l
$$

where $\varepsilon^{*}$ is the complex dielectric constant, $\Delta \varepsilon_{j}=\varepsilon_{j 0}$ $-\varepsilon_{j \infty}$, where $\varepsilon_{j 0}$ and $\varepsilon_{j \infty}$ are the low and high frequency limits of the dielectric constants, respectively, $\omega$ is the angular frequency, $\tau_{j}$ is the relaxation time, $\beta_{j}$ is a parameter characterizing the shape of the dielectric relaxation curves, and $l$ and $h$ denote the lower and higher frequency relaxation, respectively.

The relaxation strength is a measure of the number of and the orientation correlations of dipole moments along the polymer chains. The Fröhlich-Kirkwood $g$ factor given in eq 2 indicates the extent of the correlation of the dipolar motions involved in the relaxation process ${ }^{11}$;

$$
\varepsilon_{0}-\varepsilon_{\infty}=\left(\frac{3 \varepsilon_{0}}{2 \varepsilon_{0}+\varepsilon_{\infty}}\right)\left(\frac{4 \pi N}{3 k T}\right)\left(\frac{\varepsilon_{\infty}+2}{2}\right) g \mu_{0}^{2}
$$

$N$ is the number of dipoles per unit volume, $\mu_{0}$ is the dipole moment of one molecule surrounded by vacuum, $k$ is the Boltzmann constant and $T$ is the absolute temperature. The dielectric spectra obtained at $-55^{\circ} \mathrm{C}$ were found to be well fitted assuming two relaxation processes using eq 2 as shown in Figure 9. The relaxation strength is 10.36 and the shape factor is 0.34 for the main dispersion. For the higher frequency dispersion, the relaxation strength was estimated to be 0.32 assuming $\beta=1$, and then $\varepsilon_{\infty}$ was 5.35. The $g \mu_{0}^{2}$ is the effective dipole moment, and for the main dispersion the value was obtained to be $1.0 \mathrm{D}^{2}$ (the bulk density is roughly estimated to be 1 , the ether oxygen concentration would be calculated to be $0.025 \mathrm{~mol} \mathrm{ml}^{-1}$ and assuming $\mu_{0}=$ $1.20 \mathrm{D}$ for the ether oxygen dipole ${ }^{14}$ ). If the main dispersion is due to motions only related to the ether oxygen reorientation of the main chain and the side chain, the $g$ factor could be 0.7 . If dipole moments have strong correlation such as parallel alignments or anti-parallel alignments, then the factor should be either 2 or 0 . In the present case, the $g$ value indicates weak correlation of ether oxygen units in the network suggesting random reorientation of network chains.

The dipole moment of the urethane group is $3.6 \mathrm{D},{ }^{15}$ therefore it could be considered to contribute the main relaxation process. In order to evaluate dielectrically the effect of urethane group reorientation, the main dispersion curve is necessarily deconvoluted to the processes including this process. However, the main dispersion was found to be the process with the broad distribution. Information on the phenyl ring relaxation was not available from the present ${ }^{13} \mathrm{C}$ NMR spectra. The relaxation time of the dielectric main dispersion is clearly shown to correspond to the relaxation time obtained for the ethylene oxide carbon by ${ }^{13} \mathrm{C}$ NMR. This suggest that the dielectric main dispersion is mainly due to motions involving the ethylene oxide unit.

The relaxation frequency of the higher frequency process was found to be $92 \mathrm{kHz}$ at $-55^{\circ} \mathrm{C}$ and plotted in Figure 8. It has been suggested that the $\gamma$ relaxation observed in the higher molecular weight PEO samples is due to the local twisting motion of main chain in disordered regions. ${ }^{11}$ As shown in Figure 8, it could be seen that the relaxation time of the present higher frequency process would be found on the extrapolated line of the Arrhenius plots of the $\gamma$ relaxation. Since network structures are considered to have the infinite molecular weight, the PEO chains of the present study are considered to be the high molecular weight chains. The higher frequency process of this particular network could be considered to be the $\gamma$ process observed in the PEO chains.

\section{Comparison of Spectral Density Functions}

The distribution of dielectric relaxation data was obtained assuming Cole-Cole symmetrical distribution function. Finally the distribution of NMR correlation time obtained for the $T_{1}$ measurements is discussed here.

There are several mechanisms that can be contribute to spin relaxations. For the ${ }^{13} \mathrm{C}$ relaxation in the present measurements ( $T_{1}$ measurements) it could be reasonable to assume the magnetic dipolar interaction is the major factor for the mechanism because the other mechanisms can be small and ignored. ${ }^{16}$

The spectral density $J(\omega)$ is the Fourier transform of an autocorrelation function $G(\tau)$ which should reflect the nature of the local motion in bulk polymers.

$$
G(\tau)=\left\langle F(0) F^{*}(\tau)\right\rangle
$$




$$
J(\omega)=\int G(\tau) \exp (-i \omega t) \mathrm{d} t
$$

$G(t)$ is given as an exponential function from Debye's model for random motion, referred to as BPP:

$$
J(\omega)=2 \tau_{\mathrm{c}} /\left(1+\omega^{2} \tau_{\mathrm{c}}^{2}\right)
$$

Here $t_{\mathrm{c}}$ is the correlation time. Some of the spectral density functions have been proposed and used to interpret molecular dynamics of network polymers. ${ }^{17-20}$ The Cole-Cole spectral density function for NMR relaxation is given by ${ }^{20}$

$$
\begin{aligned}
J(\omega)= & (2 / \omega) \sin (\pi \beta / 2)\left\{\left(\omega \tau_{\mathrm{c}}\right)^{\beta} /\left[1+\left(\omega \tau_{\mathrm{c}}\right)^{2 \beta}\right.\right. \\
& \left.\left.+2 \cos (\pi \beta / 2)\left(\omega \tau_{\mathrm{c}}\right)^{\beta}\right]\right\}
\end{aligned}
$$

where $\omega$ is the resonance frequency. The definition of the parameter $\beta$ representing symmetrical distribution is the same as for the dielectric relaxation mentioned above. Then $T_{1}$ is given by the well-known expression ${ }^{16}$;

$$
\begin{aligned}
1 / T_{1}= & (1 / 20) \gamma_{\mathrm{C}}^{2} \gamma_{\mathrm{H}}^{2} h^{2} \sum r_{i}^{-6}\left[J\left(\omega_{\mathrm{C}}-\omega_{\mathrm{H}}\right)\right. \\
& \left.+3 J\left(\omega_{\mathrm{C}}\right)+6 J\left(\omega_{\mathrm{C}}+\omega_{\mathrm{H}}\right)\right]
\end{aligned}
$$

where $\gamma_{\mathrm{C}}$ and $\gamma_{\mathrm{H}}$ are the carbon and proton gyromagnetic ratios respectively, $r_{i}$ is the distance between the carbon and the $i$-th proton taken here as $1.09 \AA$, and $h$ is the Plank constant.

Shi et al., have shown that the temperature dependences of the ${ }^{31} \mathrm{P}$ spin-lattice relaxation times for some network polymers could be fitted using either the ColeCole distribution function or the Williams-Watts function. ${ }^{17}$ The temperature dependences of proton $T_{1}$ and $T_{1 \rho}$ in a glassy polycarbonate sample were fitted using the Williams-Watts function and the dynamic mechanical loss was simulated using the same parameters determined by NMR measurements. ${ }^{19}$ In the present study the dielectric results clearly indicate the symmetrical distribution of the relaxation time, therefore the Cole-Cole spectral density function, eq 7, could be reasonably expected to simulate NMR data.

The ${ }^{13} \mathrm{C}$ relaxation times $T_{1}$ were found to be fitted satisfactorily using eq 7, 8, and 1 . The best fitted result as shown in Figure 7 was obtained by using values $\beta=0.30$ and $B=972.4$ instead of $\beta=0.34$ and $B=$ 1159.2 for the dielectric main relaxation respectively. The parameters $\tau_{0}$ and $T_{0}$ in eq 1 are the same values as determined for the dielectric result. The distribution of the correlation times determined from NMR and that from the dielectric measurement are quite consistent in the present study. Therefore, it could be reasonable to consider that the distribution of correla- tion times of the ethylene oxide carbon is mainly due to the side chain structure of the present sample.

\section{CONCLUSION}

The dielectric main relaxation observed in the present network is considered to be motions mainly due to the ether oxygen reorientations and ${ }^{13} \mathrm{C}$ NMR results suggest the branched side chain motions could contribute the main dispersion. The higher frequency relaxation observed in the dielectric spectra could be the $\gamma$ relaxation observed in PEO chains.

\section{REFERENCES}

1. J. R. MacCallum and C. C. Vincent Ed., "Polymer Electrolyte Reviews-1", Elsevier, Amsterdam, 1987.

2. M. Watanabe, in "Solid State Ionics, Material and Application," B. V. R. Chowdari, S. Chandra, S. Singh, and P. C. Srivastava, Ed., World Scientific, Singapore, 1992.

3. K. Motogami, M. Kono, S. Mori, M. Watanabe, and N. Ogata, Electrochim. Acta, 37, 1725 (1992).

4. M. Kono, K. Furuta, S. Mori, M. Watanabe, and N. Ogata, Polym. Adv. Technol., 4, 85 (1993).

5. L. C. Dickinson, P. Morganelli, C. W. Chu, Z. Petroviz, W. J. MacKnight, and J. C. W. Chien, Macromolecules, 21, 338 (1988).

6. L. C. Dickinson, J. C. W. Chien, and W. J. MacKnight, Macromolecules, 23, 1279 (1990).

7. K. Ichikawa and W. J. MacKnight, Polymer, 33, 4693 (1992).

8. K. Ichikawa, L. C. Dickinson, W. J. MacKnight, M. Watanabe, and N. Ogata, Polymer, 33, 4699 (1992).

9. K. Ichikawa and W. J. MacKnight, Electrochim. Acta, 37, 1511 (1992).

10. S. Yoon, K. Ichikawa, W. J. MacKnight, and S. L. Hsu, Macromolecules, 28, 5063 (1995).

11. N. G. McCrum, B. E. Read, and G. Williams, "Anelastic and Dielectric Effects in Polymeric Solids," Wiley, New York, N.Y., 1969.

12. M. L. Williams, R. F. Landel, and J. D. Ferry, J. Am. Chem. Soc., 77, 3701 (1955).

13. R. H. Cole and K. S. Cole, J. Chem. Phys., 9, 341 (1941).

14. A. L. McClellan, "Tables of Experimental Dipole Moments," W. H. Freeman, San Francisco, 1963.

15. A. H. Price, in "Dielectric and Molecular Behaviour," N. E. Hill, W. H. Vaughan, A. H. Price, and M. Davis, Ed., Van Nostrand Reinhold, New York, N.Y., 1969.

16. A. A. Jones in "High Resolution NMR Spectroscopy of Synthetic Polymers in Bulk," R. A. Komoroski, Ed., VCH, Derfield Beach, Florida, 1986.

17. J.-F. Shi, L. C. Dickinson, W. J. MacKnight, and J. C. W. Chen, Macromolecules, 26, 5908 (1993).

18. C. H. Roland, Macromolecules, 27, 4242 (1994).

19. L. Lestel, P. Guegan, S. Boileau, H. Vheradame, and F. Laupretre, Macromolecules, 25, 6024 (1992).

20. M. Muller, R. Stadler, F. Kremer, and G. Williams, Macromolecules, 28, 6942 (1995).

21. P. A. Beckmann, Phys. Rep., 171, 85 (1988). 